\title{
CHARM-SYSTEM TESTS OF CPT WITH FOCUS ${ }^{a}$
}

\author{
R.W. GARDNER \\ Department of Physics, Indiana University \\ 701 East Third Street, Bloomington, IN 47405, USA \\ E-mail: rwg@indiana.edu
}

\begin{abstract}
We discuss a search for CPT violation in neutral charm meson oscillations. The data come from the Fermilab fixed-target experiment FOCUS. While flavor mixing in the charm sector is predicted to be small by the standard model, it is still possible to investigate CPT violation through study of the proper time dependence of the asymmetry in right-sign decay rates for $D^{0}$ and $\bar{D}^{0}$. Using present limits for $D^{0}-\overline{D^{0}}$ mixing we infer bounds on charm CPT violation using data from FOCUS.
\end{abstract}

\section{Introduction}

The combined symmetry of charge conjugation $(\mathrm{C})$, parity $(\mathrm{P})$, and time reversal $(\mathrm{T})$ is believed to be respected by all local, point-like, Lorentz covariant field theories, such as the standard model. However, extensions to the standard model based on string theories do not necessarily require CPT invariance, and observable effects at low-energies may be within in reach of experiments studying flavor oscillations $\mathrm{A}$ frameworll in which indirect $\mathrm{CPT}$ and $\mathrm{T}$ violating parameters appear has been developed which allows experimental investigation in neutral meson systems.

For quite some time searches for CPT violation have been made in the neutral kaon system in which particle-antiparticle mixing is large. $\mathrm{KTel}^{3}$ reports a bound on the CPT figure of merit $r_{K} \equiv\left|m_{K^{0}}-m_{\bar{K}^{0}}\right| / m_{K^{0}}<(4.5 \pm 3) \times 10^{-19}$. CPT tests in $B^{0}$ meson decay have been made by OPAI 1 at LEP, and by Belle at KEK which has recently reported $r_{B} \equiv\left|m_{B^{0}}-m_{\bar{B}^{0}}\right| / m_{B^{0}}<1.6 \times 10^{-14}$.

To date, no experimental search for CPT violation has been made in the charm quark sector. This is probably due in part to the expected suppression of $D^{0}-\bar{D}^{0}$ oscillations in the standard model, and the lack of a strong mixing signal in the experimental data. Recent mixing searches include a study of lifetime differences between charge-parity $(\mathrm{CP})$ eigenstates from FOCUS, which reported a value for the parameter $y_{C P}=(3.42 \pm 1.39 \pm 0.74) \%$. The CLEO Collaboration has reported $95 \%$ confidence level bounds on mixing parameters $x^{\prime}$ and $y^{\prime}$ (related to the usual parameters $x$ and $y$ by a strong phase shift) $(1 / 2) x^{\prime 2}<0.041 \%$ and $-5.8 \%<y^{\prime}<1 \%$. FOCUS has reported a study of the doubly Cabibbo suppressed ratio $\left(R_{D C S}\right)$ for the decay $D^{0} \rightarrow K^{+} \pi^{-}$and has

${ }^{a}$ Invited Talk given at the Second Meeting on CPT and Lorentz Symmetry, Indiana University, Bloomington, August 15-18, 2001. 
extracted a contour limit on $y^{\prime}$ (of order few \%) under varying assumptions of $R_{D C S}$ and $x^{\prime}$. The question arises - what can be learned about indirect CPT violation given the apparent smallness of mixing in the charm system? It turns out that even in the absence of a strong mixing signal one can still infer the level of CPT violation sensitivity through study of the time dependence of $D^{0}$ decays, which we show in this paper.

\section{Proper Time Asymmetry}

Time dependent decay probabilities into right-sign and wrong sign decay modes for neutral mesons which express the CPT violation have been developed in a general framework 2 For the decay of $D^{0}$ to a right-sign final state $f$ (which could be a semileptonic mode, or a Cabibbo favored hadronic mode), the time dependent decay probability is

$$
\begin{aligned}
P_{f}(t) \equiv & \left|\left\langle f|T| D^{0}(t)\right\rangle\right|^{2} \\
= & \frac{1}{2}|F|^{2} \exp \left(-\frac{\gamma}{2} t\right) \times\left[\left(1+|\xi|^{2}\right) \cosh \Delta \gamma t / 2+\left(1-|\xi|^{2}\right) \cos \Delta m t\right. \\
& -2 \operatorname{Re} \xi \sinh \Delta \gamma t / 2-2 \operatorname{Im} \xi \sin \Delta m t] .
\end{aligned}
$$

The time dependent probability for the decay of $\bar{D}^{0}$ to a right-sign final state $\bar{f}, \bar{P}_{\bar{f}}(t)$, may be obtained by replacing in the above equation $\xi \rightarrow-\xi$ and $F \rightarrow \bar{F}$. In the formula, $F$ represents the basic transition amplitude for the decay $D^{0} \rightarrow f, \Delta \gamma$ and $\Delta m$ are the differences in physical decay widths and masses for the propagating eigenstates and can be related to the usual mixing parameters $x=\Delta M / \Gamma$ and $y=\Delta \Gamma / 2 \Gamma$. The complex parameter $\xi$ controls the $\mathrm{CPT}$ violation and is seen to modify the shape of the time dependent decay probabilities. Expressions for wrong-sign decay probabilities involve both CPT and $\mathrm{T}$ violation parameters which scale the probabilities, leaving the shape unchanged. Using only right-sign decay modes, the following asymmetry can be formed,

$$
A_{C P T}(t)=\frac{\bar{P}_{\bar{f}}(t)-P(t)}{\bar{P}_{\bar{f}}(t)+P(t)},
$$

which is sensitive to CPT violating parameter $\xi$ :

$$
A_{C P T}=\frac{2 \operatorname{Re} \xi \sinh \Delta \gamma t / 2+2 \operatorname{Im} \xi \sin \Delta m t}{\left(1+|\xi|^{2}\right) \cosh \Delta \gamma t / 2+\left(1-|\xi|^{2}\right) \cos \Delta m t} .
$$

We can gain insight into the anticipated experimental sensitivity by plotting these functions with some reasonable assumptions. We use $95 \%$ confidence 
level upper bounds on the mixing parameters $x$ and $y$ of $5 \%$, which is at the upper range of the current experimental sensitivity, as discussed previously. In Fig. I (a) we plot the proper time decay probabilities for $D^{0}$ decay under the assumption of CPT violation at the level of $\operatorname{Re} \xi=5 \%, \operatorname{Im} \xi=5 \%$, which are independent parameters in the framework. One sees a CPT violation-induced wrong-sign contribution which vanishes at zero proper time and at long proper times. This causes a distortion from a purely exponential decay of a $D^{0}$ (and $\left.\bar{D}^{0}\right)$, which is then visible in the asymmetry plot, $A_{C P T}$ as shown in Fig. 11(b). Because of the small oscillation frequency and short lifetime, one sees only the start of the oscillation, growing beyond $0.3 \%$ at long proper times. Evident from Eqn. 3 is that positive values of the $\operatorname{Re} \xi$ and $\operatorname{Im} \xi$ work to oppose one another in the asymmetry in a linear fashion. This is shown in the nearly linear behavior of $A_{C P T}$ in Figs. 11(c,d) which have $\operatorname{Im} \xi=5 \%, \operatorname{Re} \xi=0$ and $\operatorname{Im} \xi=0, \operatorname{Re} \xi=5 \%$ respectively, and consequently CPT asymmetries larger by a factor of 10 at long proper times. In practice, experiments will be sensitive to either $\operatorname{Re} \xi$ or $\operatorname{Im} \xi$, but not both simultaneously.

\section{Data Analysis}

In this paper we investigate the current experimental sensitivity for a CPT violating signal using data collected by the FOCUS Collaboration during the 1996-97 fixed-target run at Fermilab. FOCUS is an upgraded version of the E687 spectrometer. Charm particles are produced by the interaction of high energy photons (average energy $\approx 180 \mathrm{GeV}$ for triggered charm states) with a segmented $\mathrm{BeO}$ target. In the target region charged particles are tracked by 16 layers of microstrip detectors. These detectors provide excellent vertex resolution. Charged particles are further tracked by a system of five multiwire proportional chambers and are momentum analyzed by two oppositely polarized large aperture dipole magnets. Particle identification is determined by three multi-cell threshold Čerenkov detectors, electromagnetic calorimeters, and muon counters.

The data analysis is as follows. We analyze the two right-sign hadronic decays $D^{0} \rightarrow K^{-} \pi^{+}$and $\bar{D}^{0} \rightarrow K^{+} \pi^{-}$. Contamination by doubly Cabibbo suppressed (wrong-sign) decay occurs but is small owing to the small branching ratio, and its effect on the right-sign signal yield estimates in this analysis is negligible. We use the soft pion from the decay $D^{*+} \rightarrow D^{0} \pi^{+}$to tag the flavor of the $D$ at production, and the kaon charge in the decay $D^{0} \rightarrow K^{-} \pi^{+}$to tag the $D$ flavor at decay. (Charge conjugates are assumed in this paper.) $D^{0} \rightarrow K^{-} \pi^{+}$events were selected by requiring a minimum detachment of the secondary (decay) vertex from the primary (production) vertex of $5 \sigma_{\ell}$. 

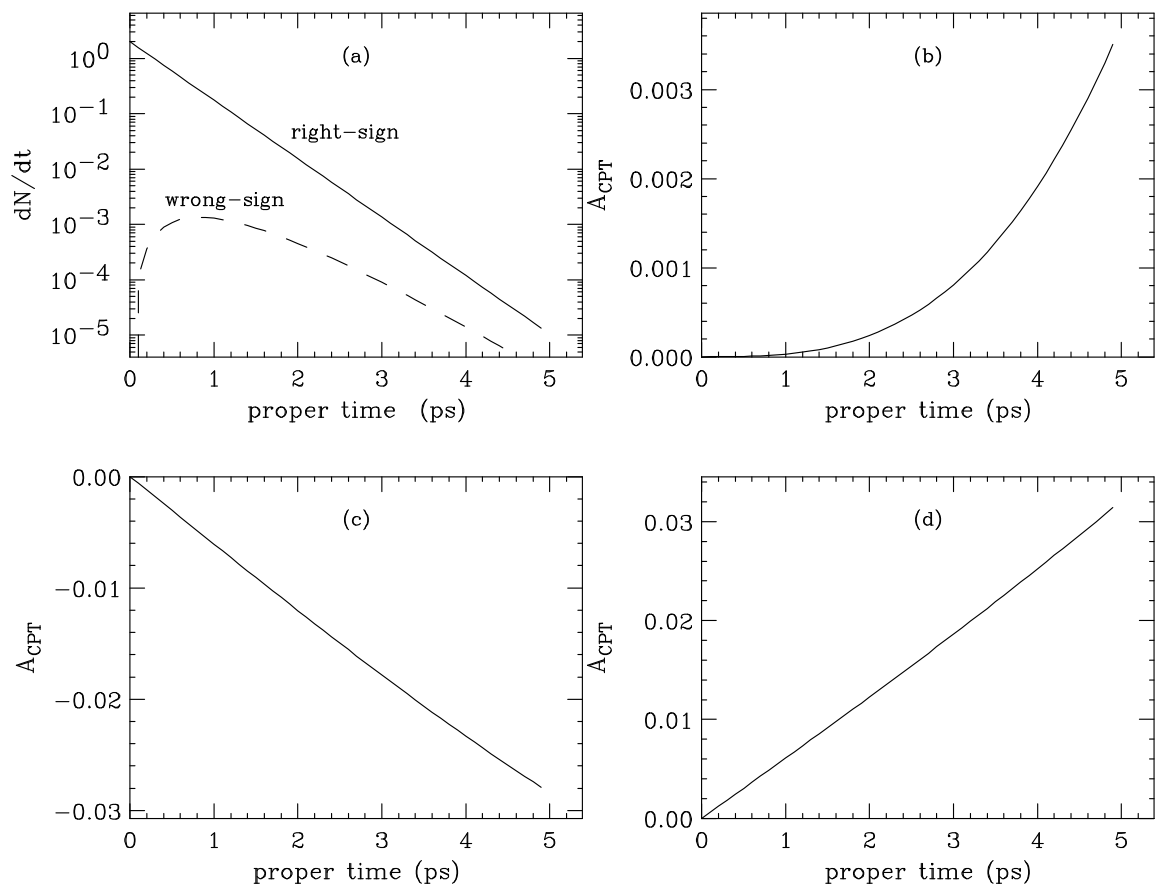

Figure 1: (a) Proper time decay probabilities for $D^{0}$ decay, right-sign (solid) and wrong-sign (dashed) and (b) $A_{C P T}$ with $\operatorname{Re} \xi=5 \%, \operatorname{Im} \xi=5 \%$. (c) $A_{C P T}$ with $\operatorname{Re} \xi=0, \operatorname{Im} \xi=5 \%$. (d) $A_{C P T}$ with $\operatorname{Re} \xi=5 \%, \operatorname{Im} \xi=0$.

The primary vertex was found using a candidate driven vertex finder which nucleated tracks about a "seed" track constructed using the secondary vertex and the reconstructed $D$ momentum vector. Both primary and secondary vertices were required to have confidence level fits of greater than $1 \%$. The $D^{*}$-tag is accomplished by requiring the $D^{*}-D^{0}$ mass difference to be less than $3 \mathrm{MeV} / \mathrm{c}^{2}$ of the nominal value

Kaons and pions were identified using the Cerenkov particle identification cuts. These cuts are based on likelihood ratios between the various stable particle hypotheses, and are computed for a given track from the observed firing response (on or off) of all cells within the track's $(\beta=1)$ Cerenkov light cone in each of three multi-cell, threshold Cerenkov counters. The probability that a given track wil fire a given cell is computed using Poisson statistics based on the predicted number of photoelectrons striking the cell's phototube under each particle identification hypothesis; the accidental firing rate of the cells, 
which depended on beam intensity, was also used. The product of all firing probabilities for all cells within the three Čerenkov cones produces a $\chi^{2}$-like variable called $W_{i} \equiv-2 \times \log$ (likelihood) where $i$ ranges over electron, pion, kaon and proton hypotheses. For the $K$ and the $\pi$ candidates we require $W_{i}$ to be no more than 4 greater than the smallest of the other three hypotheses $\left(W_{i}-\right.$ $\left.W_{\min }<4\right)$ which eliminated candidates that highly likely to be misidentified. In addition, $D^{0}$ daughters must satisfy the slightly stronger $K \pi$ separation criteria $W_{\pi}-W_{K}>0.5$ for the $K$ and $W_{K}-W_{\pi}>-2$ for the $\pi$. Doubly misidentified $D^{0} \rightarrow K^{-} \pi^{+}$is removed by imposing a hard Cerenkov cut on the sum of the two separations $\left(\left(W_{\pi}-W_{K}\right)_{K}+\left(W_{K}-W_{\pi}\right)_{\pi}>8\right)$ when the invariant mass of the track pair with the $K$ and $\pi$ hypothesis exchanged is within $4 \sigma$ of the nominal $D^{0}$ mass. Fig. 2 2 shows the invariant mass distribution for two $D^{*}$-tagged, right-sign decays $D^{0} \rightarrow K^{-} \pi^{+}$and $\bar{D}^{0} \rightarrow K^{+} \pi^{-}$. Approximately 49700 signal events were used in the analysis described below.

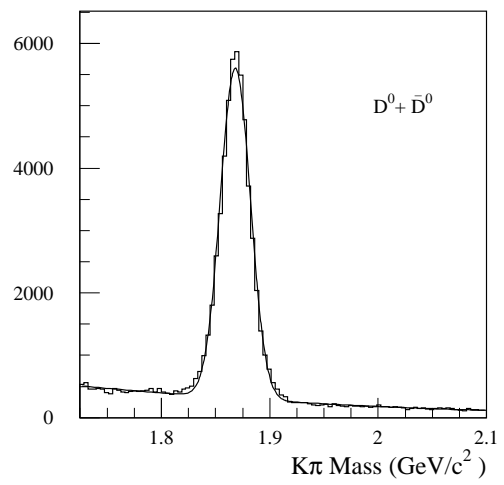

Figure 2: Invariant mass distribution for the sum of $D^{0}$ and $\hat{D}^{0}$ right-sign decay candidates.

The reduced proper time is a traditional lifetime variable used in fixedtarget experiments which use the detachment between the primary and secondary vertex as their principal tool in reducing non-charm background. The reduced proper time is defined by $t^{\prime}=\left(\ell-N \sigma_{\ell}\right) /(\beta \gamma c)$ where $\ell$ is the distance between the primary and secondary vertex, $\sigma_{\ell}$ is the resolution on $\ell$, and $N$ is the minimum detachment cut required to tag the charmed particle through its lifetime. Fig. 3 shows reduced proper time distributions for the two right-sign decays $D^{0} \rightarrow K^{-} \pi^{+}$and $\bar{D}^{0} \rightarrow K^{+} \pi^{-}$.

We plot the difference in right-sign events between $\bar{D}^{0}$ and $D^{0}$ in bins of reduced proper time $t^{\prime}$ in Fig. 4. For each data point, the background subtracted yields of right-sign $D^{0}$ and $D^{0}$ are used in forming the ratio. In the absence 

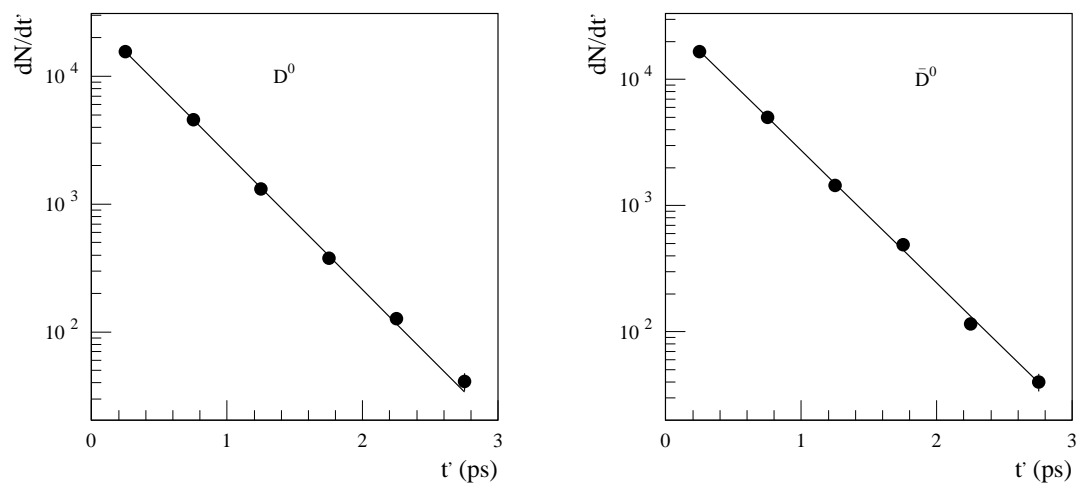

Figure 3: Background subtracted reduced proper time distributions for $D^{0}$ and $\overline{D^{0}}$.

of detector acceptance corrections, this is equivalent to $A_{C P T}$ as defined in Eqn. 2. Monte Carlo studies showed that detector and resolution effects cause less than $5 \%$ changes in the $t^{\prime}$ distribution as measured by deviations from a pure exponential decay. $A_{C P T}$ is additionally insensitive to detector effects since they tend to cancel in the ratio.

Because of the QCD production mechanism for photoproduced charm mesons, more $\overline{D^{0}}$ than $D^{0}$ are produced in the FOCUS data sample. This has been previously investigated in photoproduction by E687, in which the production asymmetries were studied in the context of a string fragmentation model 10 The effect in the $A_{C P T}$ distribution is to add a constant, production-related offset, which can be accounted for in fits to the asymmetry distributions.

We infer the CPT sensitivity for a fixed set of experiment bounds on mixing without CPT violation. The $A_{C P T}$ data in Fig. 1 are fit using the time dependent probability functions for $\bar{D}^{0}$ and $D^{0}$, as defined by Eq. 1 for the case of $D^{0}$ decay. The allowed fit parameters are a constant production asymmetry parameter and $\operatorname{Re} \xi$ (fits with $\operatorname{Im} \xi$ gave similar results for sensitivity). In the fit shown we used values for the $x$ and $y$ mixing parameters of $5 \%$, and infer one standard deviation errors on either $\operatorname{Re} \xi$ or $\operatorname{Im} \xi$ of approximately $10 \%$, corresponding to $95 \%$ confidence level upper bounds of roughly $25 \%$.

In the CPT and Lorentz violating extension to the standard model 11 the CPT violating parameters may depend on lab momentum, orientation, and sidereal time12 2 They are expressed in terms of Lorentz violating coupling coefficients which depend on the flavor of the valence quark states. (For this reason, CPT violation in the $K, D$, and $B$ systems need not be same.) In the 

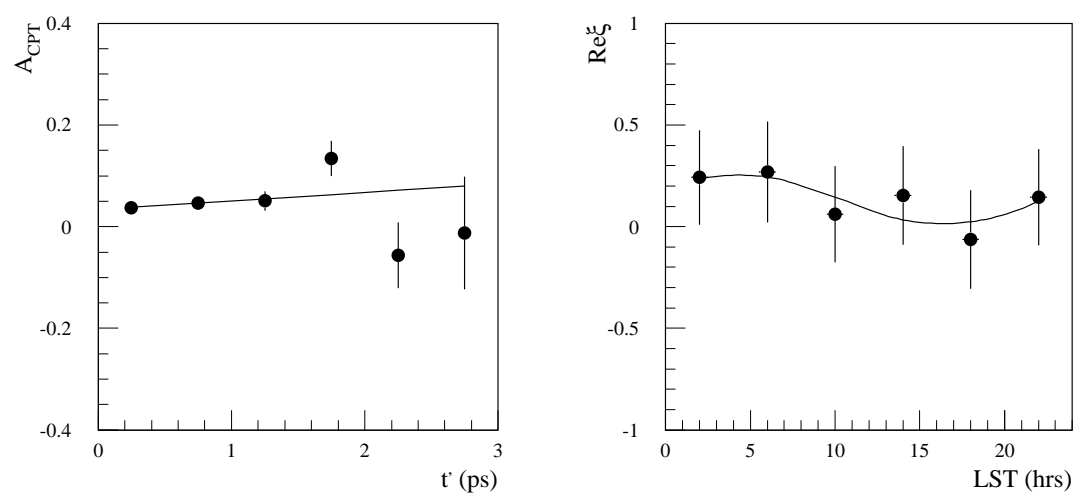

Figure 4: Asymmetry as a function of reduced proper time (left). At the right is plotted the variation of the fitted CPT parameter Re $\xi$ as a function of local sidereal hour.

case of FOCUS, a forward, fixed-target spectrometer, the $\xi$ parameter assumes the following form

$$
\begin{aligned}
\xi(\hat{t}, p)= & \frac{\gamma(p)}{\Delta \lambda}\left[\Delta a_{0}+\beta \Delta a_{Z} \cos \chi\right. \\
& \left.+\beta \sin \chi\left(\Delta a_{Y} \sin \Omega \hat{t}+\Delta a_{X} \cos \Omega \hat{t}\right)\right],
\end{aligned}
$$

where $\Omega$ and $\hat{t}$ are the sidereal frequency and time respectively, and $X, Y, Z$ are non-rotating coordinates with $Z$ aligned with the Earth's rotation axis. Our sensitivity quoted above should then be regarded as that for $|\bar{\xi}|$ averaged over momentum and sidereal time. From this quantity one can infer the sensitivity for the Lorentz violating charm and $\bar{u}$ quark coefficients: $\Delta a_{0}+0.6 \times \Delta a_{Z}<$ $10^{-15} \mathrm{GeV}$, where we have used 53 degrees as the angle between the FOCUS spectrometer axis and the Earth's pole.

We searched for a sidereal dependence, which would be sensitive to coefficients $\Delta a_{X}$ and $\Delta a_{Y}$, by dividing our data sample into four-hour bins in sidereal time and repeating our fits. The resulting distribution, shown also in Fig. 4 , can be fit using Eq. fabove to yield bounds on $\Delta a_{X}$ and $\Delta a_{Y}$ of similar sensitivity.

\section{Conclusions}

We have investigated the sensitivity to a CPT violating signal in neutral charm meson oscillations, the first search of its kind in the charm system. Our preliminary study using FOCUS data indicates $95 \%$ confidence level upper bounds 
on charm CPT violation parameters $\operatorname{Re} \xi$ and $\operatorname{Im} \xi$ of approximately $25 \%$, corresponding to bounds on the CPT and Lorentz violating charm coefficients of $<10^{-15} \mathrm{GeV}$.

\section{Acknowledgments}

We wish to acknowledge the assistance of the staffs of the Fermi National Accelerator Laboratory, the INFN of Italy, and the physics departments of the collaborating institutions. The research was supported in part by the U.S. National Science Foundation, the U.S. Department of Energy, the Italian Instituto Nazionale di Fisica Nucleare and Ministero dell'Universita della Ricerca Scientifica e Tecnologica, the Brazilian Conselho Nacional de Desenvolvimento Cientfico e Tecnologico, CONCAyT-Mexico, the Korean Ministry of Education, and the Korean Science and Engineering Foundation.

\section{References}

1. V.A. Kostelecký, R. Potting, Phys. Rev. D 51, 3923 (1995).

2. V.A. Kostelecký, Phys. Rev. D 64, 076001 (2001).

3. Y.B. Hsiung for the $\mathrm{KTeV}$ Collaboration, Proc. QCD Euroconference 99, Montpellier, France, July 7-13, 1999. Also available as FERMILABConf-99/338-E (KTeV).

4. OPAL Collaboration (K. Ackerstaff et al.), Z.Phys.C76:401-415,1997.

5. Belle Collaboration (K. Abe, et al.), Phys. Rev. Lett. 86, 3228 (2001).

6. FOCUS Collaboration, J. Link et al., Phys. Lett. B 485, 62 (2000).

7. FOCUS Collaboration, J. Link et al., Phys. Rev. Lett. 86, 2955 (2001).

8. CLEO Collaboration, R. Godang et al., Phys. Rev. Lett. 84, 5038 (2000).

9. Review of Particle Properties, D.E. Groom et al., Eur. Phys. J. C 15, 1 (2000).

10. E687 Collaboration, P.L. Frabetti et al., Phys. Lett. B 370, 222 (1996).

11. D. Colladay and V.A. Kostelecký, Phys. Rev. D 55, 6760 (1997); Phys. Rev. D 58, 116002 (1998).

12. V.A. Kostelecký, Phys. Rev. Lett. 80, 1818 (1998). 